Sitientibus Série Ciências Físicas 02: 1-6 (2006)

\title{
Efeito da Desordem em Excitações Magnéticas Elementares
}

\author{
Effect of Disorder in Elementary Magnetic Excitations
}

\author{
Elton M. Nascimento* \\ Instituto de Física - UFAL \\ Campus A.C. Simões, Km 14, BR 104 \\ Maceió - AL - 57072-970 \\ Antônio Delson C. de Jesus ${ }^{\dagger}$ \\ Departamento de Física - UEFS \\ Campus Universitário, s/n, Km 03, BR 116 \\ Feira de Santana - BA - 44031-460
}

\begin{abstract}
A inclusão da desordem nos diversos sistemas físicos tem trazido relevantes resultados nos últimos anos no estudo das propriedades dos materiais. Devido à forte relação entre o modelo de Anderson e o de Heisenberg, para as excitações magnéticas elementares, é esperado que a desordem excerça um papel semelhante para ambos os casos, desta forma, munidos de recursos computacionais mais avançados, refizemos os cálculos para investigar o papel da desordem nos auto-estados de 1-magnon. Os resultados já encontrados para o caso eletrônico nos motivaram a investigar, também, a inclusão de correlações na distribuição de desordem para o caso magnético. Nossos resultados confirmaram as expectativas, ou seja, a inclusão de desordem nas constantes de interação de troca entre os pares de spin faz com que os seus auto-estados se limitem à uma região finita da cadeia. Já com a inclusão da correlação de longo alcance, esta tendência sofre uma mudança para um grau da correlação $\alpha>1.0$. Neste caso, surge uma banda de auto-estado estendido em $\epsilon<2,5$.
\end{abstract}

Palavras-chave: Magnon, Desordem, Auto-Estados, Correlação, Cadeia Linear.

In many physical systems the inclusion of disorder effect has been brought important results in the last years when we study the proprieties of materials. Due to strong relation between the Anderson and Heisenberg models when we consider the elementary magnetic excitations and expecting the disorder plays a similar role in these both cases we carry out calculations to investigate the role of disorder in the 1-magnon eigenstate, using to do this new computational resources. The relevant results already found in the electronic case have stimulated an investigation to include the correlations in the disorder distribution for the magnetic case. Our results have confirmed our expectations, i.e., the inclusion of disorder effect through the exchange interaction parameters among pairs of spin turn the eigenstates to be limited themselves to a finite region in the chain. In the other side case the inclusion of correlation of long range, this tendency suffers a change for a degree of correlation $\alpha>1.0$. In this last case we observe the appearance of a of extended eigenstate for $\epsilon<2,5$.

Keywords: Magnon, Disorder, Eigenstates, Correlation, Linear Chain.

\section{INTRODUÇÃO}

O entendimento das propriedades dos materiais sempre esteve entre os principais enig-

\footnotetext{
*Endereço Eletrônico: eltonmalta@bol.com.br

${ }^{\dagger}$ Endereço Eletrônico: adj@uefs.br
}

mas da ciência, já que o conhecimento de tais propriedades, além de sanar a própria curiosidade inerente à sociedade, proporciona avanços tecnológicos e soluções para os problemas do mundo em que vivemos.

Em meados do século XX, a descoberta de novas técnicas matemáticas possibilitou o estudo teórico dos chamados "materiais im- 
puros", ou seja, que apresentam desordem na sua formação, como uma "mistura" de átomos, onde não existe ordem na posição de seus elementos. Viu-se, então, que a inclusão da desordem proporcionava mudanças notáveis nas propriedades da matéria. Em 1979, P. W. Anderson et al. [1], utilizando um modelo que permitiu estudar os efeitos da desordem sobre a função de onda eletrônica, verificaram que, para sistemas com dimensão menor que 2 , a função de onda sempre se encontrava localizada em uma região finita do sistema, independente do grau de desordem; é a chamada fase isolante. Já para sistemas de dimensões maiores que 2, a propriedade de localização da função de onda passa a depender do grau de desordem do sistema, ou seja, no limite de forte desordem, o modelo de Anderson prevê que os estados eletrônicos apresentem fase isolante, já no limite de fraca desordem estes estados deixam de ser localizados e o elétron passa a apresentar probabilidade de ser encontrado em qualquer sítio do sistema; é a chamada fase metálica. Este fenômeno é a chamada Transição MetalIsolante ou Transição de Anderson.

Este modelo de escala foi quebrado, apenas, com a inclusão de correlações na distribuição de desordem do material. P. Dean [2] introduziu correlações de curto alcance na distribuição de desordem em cadeias diatômicas e detectou picos na distribuição de frequências, associados à frequência dos modos de impureza, característica não encontrada em redes ordenadas. Fenômenos ainda mais curiosos foram encontrados, como o obtido por Dunlap, Wu e Phillips [3] ao investigarem uma cadeia do tipo "liga binária". Neste tipo de sistema, as energias dos sítios assumem apenas os valores $\epsilon_{A}$ ou $\epsilon_{B}$, aleatoriamente distribuídos, e os sítios com energia $\epsilon_{A}$ sempre aparecem aos pares. Foi verificada a possibilidade de existência de estados ressonantes, onde a função de onda é delocalizada. Tais resultados foram comprovados experimentalmente por Bellani et al. [4] em super-redes de GaAs e AlGaAs.

As seqüências desordenadas, com correlação de longo alcance, não apresentam comprimento de escala característico [5] e sua densidade espectral é aproximadamente uma lei de potência da forma $S(k)=1 / k^{\alpha}$, onde $\alpha$ é um parâmetro que controla o grau de correlação, tal que, para $\alpha=0$, o sistema não possui correlação e as variáveis aleatórias são completamente independentes umas das outras. Em 1998, Moura e Lyra [6] aplicaram a correlação de longo alcance aos elementos da diagonal do Hamiltoniano que descreve o modelo de Anderson. Isto representava, fisicamente, uma desordem apenas nos potenciais de cada sítio da rede. Utilizando um formalismo de grupo de renormalização, eles mostraram que este sistema pode exibir uma fase de estados estendidos no centro da banda, se $\alpha>2$. Eles obtiveram resultados semelhantes para o caso em que a desordem se aplicava aos termos de hopping do Hamiltoniano sendo que, desta vez, a transição acontecia para $\alpha>1$. Pela primeira vez, uma verdadeira transição metal-isolante foi encontrada em sistemas 1D desordenados.

Devido a esta impressionante característica, outros sistemas foram estudados na presença de correlações de longo alcance, como cadeias de DNA $[7,8]$ e cadeias harmônicas com massas randômicas correlacionadas [9]. Para todos estes casos, o mesmo fenômeno, obtido no caso eletrônico, também foi encontrado.

Neste trabalho tratamos das excitações de 1-magnon, ou seja, a partir do estado fundamental do ferromagnetismo um único spin é induzido a mudar de orientação. Devido às interações de troca entre os spins vizinhos, este desvio se propaga pela rede. O entendimento das ondas de spin tem papel importante na descrição física das propriedades da matéria. É comprovado experimentalmente, por exemplo, que as ondas de spin contribuem para o calor específico do EuS [10]. Pelo fato do problema magnético ser mapeável, através do modelo de Anderson, é esperado que os resultados obtidos sobre os efeitos da desordem no problema eletrônico se repita aqui.

\section{MODELO E FORMALISMO}

Tratamos de uma cadeia ferromagnética unidimensional de spins $S=1 / 2$, com in- 
teração de troca isotrópica entre primeiros vizinhos do tipo Heisenberg, cujo hamiltoniano é dado por:

$$
H=-\sum_{n=1}^{N} J_{n, n+1} \vec{S}_{n} \cdot \vec{S}_{n+1},
$$

onde $J_{n, n+1}$ representa a constante de interação de troca entre os spins dos sítios $n$ e $n+1$. Para a ordem ferromagnética, esta constante deve ser sempre positiva.

Para representar os auto-estados de 1magnon, escrevemos a função de onda $|\psi\rangle$ como uma combinação linear dos vetores $\left|\phi_{n}\right\rangle$. Conforme abaixo:

$$
\left|\psi>=\sum_{n=1}^{N} b_{n}\right| \phi_{n}>,
$$

os vetores da base $\left(\mid \phi_{n}>\right)$ representam estados nos quais o sistema se encontra. No estado fundamental do ferromagnetismo todos os spins apontam na mesma direção, porém o spin do sítio $n$ tem sua orientação alterada. Supondo que no estado fundamental os spins apontem para o sentido positivo do eixo $z$, e este estado seja representado pelo vetor $\left|\phi_{0}\right\rangle$, podemos escrever os vetores da base como sendo:

$$
\left|\phi_{n}>=\overrightarrow{S_{n}^{-}}\right| \phi_{0}>\text {, }
$$

onde $\overrightarrow{S_{n}^{-}}$é um operador que muda a orientação do spin localizado no sítio $n$. Resolvendo a equação de Schrodinger, independente do tempo, nesta representação e tomando $\hbar=1$, obtivemos a seguite relação para os coeficientes $b_{n}$.

$$
\begin{aligned}
\left(J_{n, n+1}+J_{n-1, n}\right) & b_{n}+J_{n, n+1} b_{n+1} \\
& +J_{n-1, n} b_{n-1}=2 \epsilon b_{n},(4)
\end{aligned}
$$

onde $\epsilon$ é a energia de excitação do sistema.

A desordem será introduzida na distribuição dos valores das constantes de troca $\left(J_{n}\right)$. O sistema de relações resultante é calculado numericamente através de uma diagonalização direta, onde se obtém as energias e suas respectivas auto-funções.
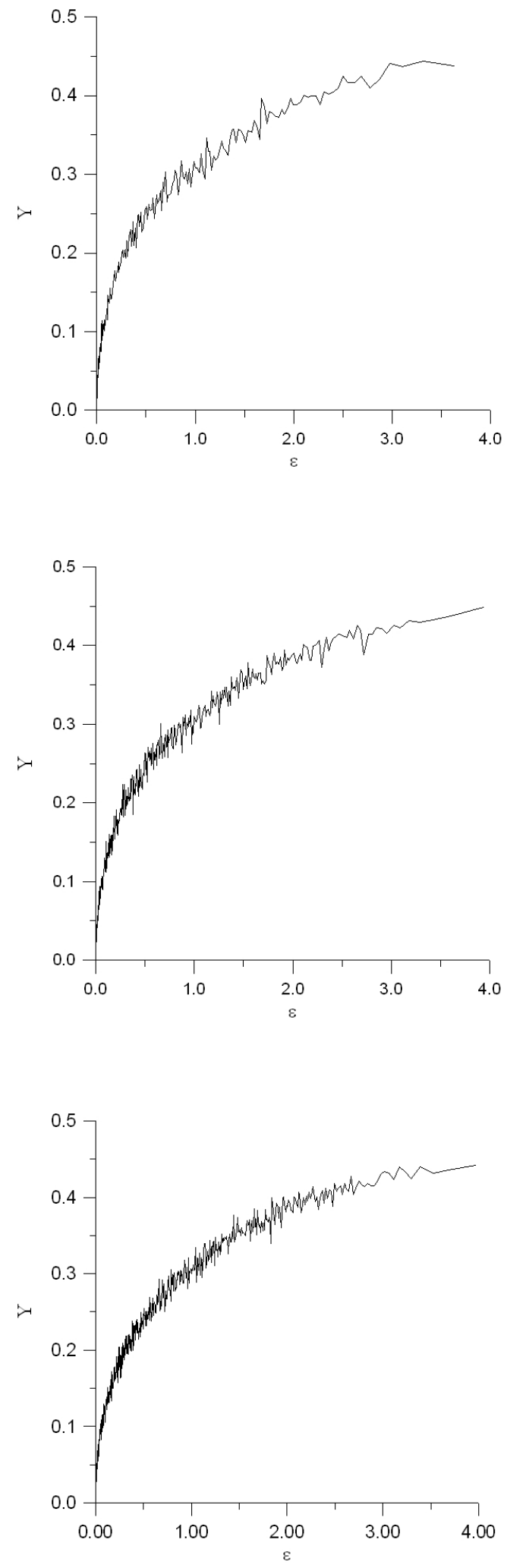

FIGURA 1: Inverso da função de participação dos auto-estados de 1-magnon para cadeia com $\mathrm{N}=200$, 400 e 600 (de cima para baixo). Tendência de localização com o aumento da energia de excitação. 

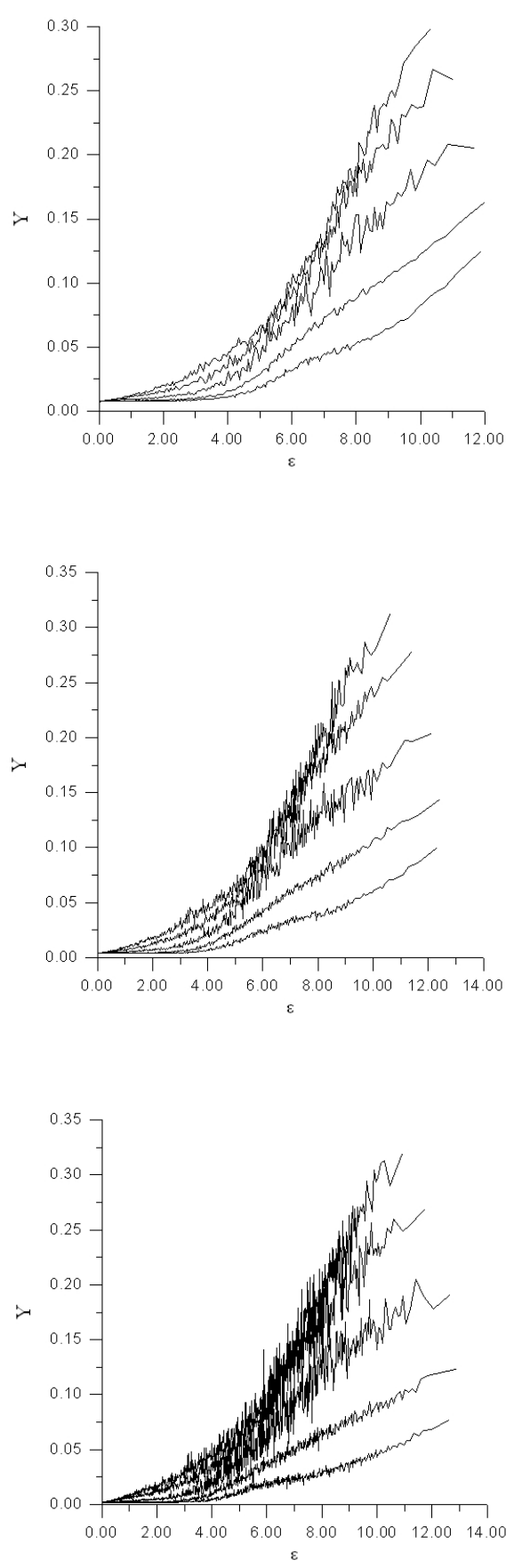

FIGURA 2: Inverso da função de participação dos auto-estados de 1-magnon com inclusão de correlações de longo alcance. Para cada gráfico temos, de baixo para cima, $\alpha=2,0 ; 1,5 ; 1,0$ e 0,5 . Do gráfico de cima para o de baixo temos $\mathrm{N}=200,400$ e 800. A correlação faz surgir uma fase de estados estendidos para $\epsilon<2,5$ à partir de $\alpha>1,0$.

A fim de estimar as propriedades de localização da função de onda de 1-magnon, na presença da desordem, foi calculada o inverso da função de participação (Y), dada pela expressão:

$$
Y=\frac{\sum_{n=1}^{N} b_{n}^{4}}{\left(\sum_{n=1}^{N} b_{n}^{2}\right)^{2}} .
$$

Como a fução de onda é normalizada, o denominador tem valor unitário. A função de participação tem relação com o número de sítios que participam da função de onda, ou seja, para auto-estados cada vez mais localizados, o inverso da função de participação apresenta valor cada vez maior.

Devido às limitações computacionais, foram calculadas cadeias com tamanho máximo de $N=1000$ e com um número de 100 realizações, para cada tamanho de cadeia. Em cada realização tem-se uma nova distribuição de desordem, sendo os resultados mostrados aqui numa média aritimética entre todas as realizações.

\section{RESULTADOS}

\section{A. Desordem sem Correlação}

A Figura 1 mostra os resultados para o caso em que os valores das constantes de interação de troca obedecem a uma distribuião uniforme de probabilidades, com média 0 e variância 1 , onde foi tomado o módulo dos valores negativos e excluídos os valores nulos. De cima para baixo temos $N=200,400$ e 600 . Em todas os tamanhos de cadeia podemos observar a mesma tendência de localização com o aumento da energia de excitação.

Este resultado está de acordo com nossas previsões, já que no modelo original de Anderson, para qualquer grau de desordem, em sistemas com dimensão menor que 2 , os autoestados eletrônicos são sempre localizados. A única diferença está no fato de que os autoestados de 1-magnon, com energia de excitação zero, se apresentam estendidos.

Através de uma linearização dos gráficos da 
Figura 1, podemos verificar uma lei de potência para a função de participação do tipo $Y \propto \epsilon^{\beta}$, onde foi verificado que, para cada tamanho de cadeia, o expoente $\beta$ apresenta um valor diferente, conforme consta na Tabela 1.

\begin{tabular}{l|l}
$\mathrm{N}$ & $\beta$ \\
\hline 100 & 0,33946 \\
200 & 0,34802 \\
400 & 0,35459 \\
600 & 0,37592 \\
1000 & 0,39568
\end{tabular}

TABELA I: Número de sítios versus o expoente da lei de potência.

A fim de se obter um expoente para uma cadeia de dimensões macroscópicas, traçamos o gráfico de $\beta \times 1 / N$ (Figura 3 ), pois assim, através de uma extrapolação da reta na direção de $1 / N \rightarrow 0$, estimamos que $\beta_{\infty}=0,463749$.

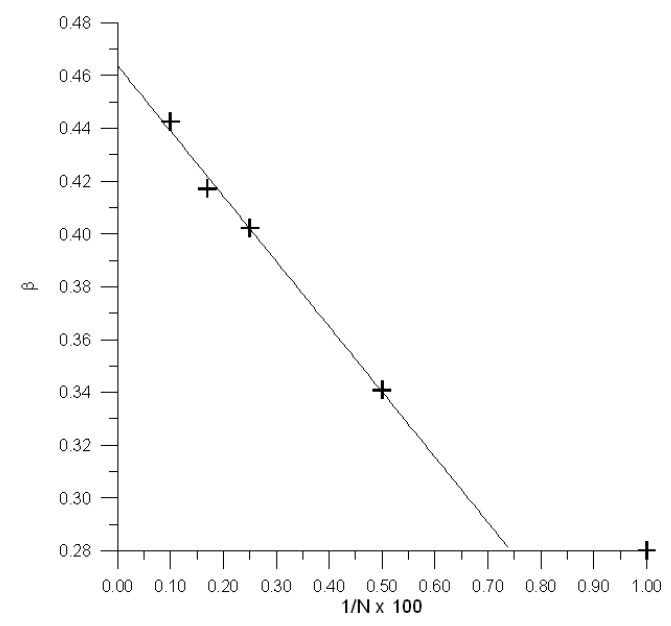

FIGURA 3: Estimativa para $\beta_{\infty}=0,463749$ através da extrapolação da reta $\beta \times 1 / N$ quando $1 / N \rightarrow 0$.

\section{B. Desordem com Correlações de Longo Alcance}

Neste ponto, o valor das constantes de troca mantém seu caráter aleatório. No entanto, os valores da distribuição apresentam uma correlação com seu antecessor. Esta distribuição pode ser obtida fazendo com que os valores de
$J_{n}$ obedeçam uma distribuição tal que descreva o traço de um movimento Browniano com densidade espectral do tipo $S(k) \propto 1 / k^{\alpha}$. Esta distribuição pode ser obtida pela expressão

$$
\begin{aligned}
J_{n, n+1} & =\sum_{k=1}^{N / 2}\left[k^{-\alpha}\left(\frac{2 \pi}{N}\right)^{(1-\alpha)}\right]^{1 / 2} \times \\
& \times \cos \left(\frac{2 \pi n k}{N}+\phi_{k}\right),
\end{aligned}
$$

onde $\phi_{k}$ representa fases aleatórias que variam de 0 à $2 \pi$. Desta forma, o valor de $\alpha$ controla o grau de correlaçõ da distribuição, tal que, para $\alpha=0$, reproduzimos uma distribuição uniforme de desordem, como descrito anteriormente. Os resultados para o inverso da função de participação são mostrados na Figura 2.

Cada gráfico representa um tamanho de cadeia e para cada tamanho de cadeia foi calculado os resultados para $\alpha=0,5 ; 1.0 ; 1,5$ e 2,0 . Para qualquer um dos tamanhos observase a mesma tendência. Nas cadeias com grau de correlação iguais a 0,5 e 1,0 a participação cresce com o aumento da energia de excitação, como ocorrido para uma desordem uniforme e não correlacionada. Para $\alpha=1,5$ e 2, 0 notase que, na região de baixa energia, $\epsilon<2,5$, a função de participação se mantém constante e igual ao menor valor possível, para cada tamanho de cadeia, ou seja, $Y_{\min }=1 / N$. Isto implica que, para um grau de desordem $\alpha>$ 1,0 , os auto-estados, cuja energia de excitação se encontre dentro desta banda de energia, se apresentam estendidos ao longo da cadeia, ou seja, a excitação magnética apresenta probabilidade de ser encontrada em qualquer sitio da cadeia. Mais uma vez os resultados obtidos, para o modelo de Anderson, se repetem para o modelo de Heisenberg.

\section{CONCLUSÕES}

Nossos resultados confirmaram nossas expectativas, ou seja, o fenômeno da localização dos auto-estados induzidos pela inclusão da desordem se aplica também às excitações de 1magnon. Através da análise da função de par- 
ticipação, vimos que, para uma distribuição de desordem uniforme e sem correlações, os autoestados tendem a serem mais localizados com o aumento da energia de excitação. Com a inclusão de correlações de longo alcance, este fenômeno apresenta uma variante, ou seja, para um grau de correlação $\alpha>1,0$, a correlação passa a induzir o surgimento de uma fase de auto-estados estendidos para $\epsilon<2,5$. Apesar desta pesquisa ter feito parte de um trabalho de conclusão de curso de graduação, os resultdos para desordem, com correlação de longo alcance, foram originais e se encontram na íntegra em [11].

\section{AGRADECIMENTOS}

Gostaria de agradecer a contribuição neste trabalho do Prof. Dr. Marcelo Leite Lyra do Instituto de Física da UFAL e aos órgãos de fomento à pesquisa da UEFS.
[1] E. Abrahams, P.W. Anderson, D.C. Licciardello, T. V. Ramakrishnann. Phys. Rev. Lett. 42, 673 (1979).

[2] P. Dean. Proc. Roy. Soc. 254, 507 (1960).

[3] D.H. Dunlap, H-L Wu, P.W. Phillips. Phys. Rev. Lett. 65, 1 (1990).

[4] V. Bellani, E. Diez, R. Hey, L. Toni, L. Torricone, G.B. Parravicini, R. Domínguez-Adame, R. Gómez-Alcalá. Phys. Rev. Lett. 82, 10 (1999).

[5] M. Paczuski, S. Maslov, P. Bak. Phys. Rev. E 53, 414 (1996). (E as referências nelas contidas).

[6] F.A.B.F. de Moura, M.L. Lyra. Phys. Rev.
Lett. 81, 3735 (1998).

[7] H. Yamada. Phys. Lett. A 332, 65 (2004).

[8] H. Yamada. Int. J. Mod. Phys. B 18, (12) 1697 (2004).

[9] F.A.B.F. de Moura, M.D. Coutinho, E.P. Raposo, M.L. Lyra. Phys. Rev. B 68, (1) 012202 (2003).

[10] D.C. McCollum, J. Callaway. Phys. Rev. Lett. 9, (9) 376 (1962).

[11] R.P.A. Lima, M.L. Lyra, E.M. Nascimento, A.D. de Jesus. Phys. Rev. B 65, 104416 (2002). 